\title{
Combination of an E-Nose and an E-Tongue for Adulteration Detection of Minced Mutton Mixed with Pork
}

\author{
Xiaojing Tian, ${ }^{1,2}$ Jun Wang $\mathbb{D}^{1},{ }^{1}$ Zhongren $\mathrm{Ma}^{2}{ }^{2}$ Mingsheng $\mathrm{Li}^{2}{ }^{2}$ and Zhenbo Wei ${ }^{1}$ \\ ${ }^{1}$ Department of Biosystems Engineering, Zhejiang University, 886 Yuhangtang Road, Hangzhou 300058, China \\ ${ }^{2}$ College of Life Science and Engineering, Northwest Minzu University, Lanzhou 730024, China \\ Correspondence should be addressed to Jun Wang; jwang@zju.edu.cn
}

Received 28 September 2018; Revised 17 December 2018; Accepted 21 February 2019; Published 16 April 2019

Guest Editor: José M. Díaz-Cruz

Copyright (C) 2019 Xiaojing Tian et al. This is an open access article distributed under the Creative Commons Attribution License, which permits unrestricted use, distribution, and reproduction in any medium, provided the original work is properly cited.

\begin{abstract}
An E-panel, comprising an electronic nose (E-nose) and an electronic tongue (E-tongue), was used to distinguish the organoleptic characteristics of minced mutton adulterated with different proportions of pork. Meanwhile, the normalization, stepwise linear discriminant analysis (step-LDA), and principle component analysis were employed to merge the data matrix of E-nose and E-tongue. The discrimination results were evaluated and compared by canonical discriminant analysis (CDA) and Bayesian discriminant analysis (BAD). It was shown that the capability of discrimination of the combined system (classification error $0 \%$ $1.67 \%$ ) was superior or equable to that obtained with the two instruments separately, and E-tongue system (classification error for E-tongue 0 2.5\%) obtained higher accuracy than E-nose (classification error $0.83 \% \sim 10.83 \%$ for E-nose). For the combined system, the combination of extracted data of 6 PCs of E-nose and 5 PCs of E-tongue was proved to be the most effective method. In order to predict the pork proportion in adulterated mutton, multiple linear regression (MLR), partial least square analysis (PLS), and backpropagation neural network (BPNN) regression models were used, and the results were compared, aiming at building effective predictive models. Good correlations were found between the signals obtained from E-tongue, E-nose, and fusion data of E-nose and E-tongue and proportions of pork in minced mutton with correlation coefficients higher than 0.90 in the calibration and validation data sets. And BPNN was proved to be the most effective method for the prediction of pork proportions with $R^{2}$ higher than 0.97 both for the calibration and validation data set. These results indicated that integration of E-nose and E-tongue could be a useful tool for the detection of mutton adulteration.
\end{abstract}

\section{Introduction}

There is a consistent growth in the demand for meat with high nutritional value, enriched with several vitamins, minerals, and essential polyunsaturated fatty acids and low in fat and cholesterol [1]. However, it is noted that processed mutton products have been adulterated with other types of cheaper meat, including vegetarian meat, to increase its profit $[2,3]$. The worse is that pork back fat has been added to these processed mutton products in some cases [1]. In addition, the horse-meat scandal in 2013 and the frequently reported mutton adulteration have seized the attention of consumers all over the world. Reliable methods to detect mutton adulteration based on E-tongue were established [4], and it is necessary to establish fast and reliable methods to verify meat adulteration in different viewpoints.

For meat species and meat adulteration detection, many studies were reported. The methods used included molecular biology-based methods [5, 6], enzymatic immunological methods [7], chromatographic methods [8, 9], spectroscopy methods [10-12], and electronic sensory evaluation $[9,13]$.

With the advantages of small amount of the sample required, speed, simplicity, high sensitivity, and good correlation with data from sensory analyses, the use of the electronic sensory evaluation of E-nose and E-tongue to evaluate the quality of meat has become more popular. E-tongue was shown to be able to discriminate different 
species of chicken $[14,15]$ and fishes [16]. E-nose was shown to be useful in meat products differentiation and authenticity assessment [9] and the identification and differentiation of pork for halal authentication [17] and pork adulteration in mutton [13].

However, the use of E-nose or E-tongue could only reflect one aspect for the sample, which may cause inaccuracy of the classification. By fusion of E-nose or E-tongue responses, the discrimination of meat adulteration could be improved by giving the overall sensory evaluation of meat, closer to human judgment. The combination of E-nose and E-tongue had been reported in the researches in geographical origin identification of potato creams [18] and virgin olive oil [19-21]; varieties and grade level discrimination of Chinese green tea [22], black tea [23], red wine [24], and fruit juice $[25,26]$; cultivars discrimination and characterisation of Perilla frutescens [27] and species differentiation of coffee [28]; freshness evaluation of wine [29] and milk [30]; quality differentiation [31]; and authenticity assessment [32]. These studies provided references for the in-depth study of the food quality inspection of E-nose and E-tongue.

For the fusion of E-nose or E-tongue responses, three ways [33] of abstraction were reported. For the original sensor fusion method, known as the low-level fusion, the data from E-nose and E-tongue were simply concatenated as the input data set for model construction, with the number of rows equal to the number of samples and the number of columns equal to the total number of signals from E-nose and E-tongue. Sometimes, the combined data set faced with normalization or extraction of the eigenvalue. However, for fusion of extracted features, named midlevel fusion, feature extraction methods were applied to each data source before the extracted features are combined. The commonly used selection feature methods are analysis of variance, principle component analysis, stepwise discriminant analysis, Bayes discriminant analysis, and so on. Fusion of extracted features is more popular, as the redundant information and multiple collinearity problem of data set were eliminated, and the data dimensions were reduced. For the high-level fusion, separate models were built for each data set of E-nose and E-tongue, respectively, and then the models were combined to give fused responses. It is the fusion of results.

Of the three levels of data fusion, low and midlevel fusion are the most used. Low-level fusion is the first attempt approach, but when data are very different in size or scale, the more tuneable midlevel fusion can yield better results [34]. High-level fusion, instead, is the least used approach, as the best classification or prediction model had to be determined to ensure that the fused results are better than individual models.

In this study, low-level fusion and midlevel fusion methods were studied for the fusion of E-nose or E-tongue data in the discrimination of mutton authentication. The aim of this study was to evaluate the effectiveness of data fusion methods for E-nose and E-tongue in the authentication of mutton according to its proportion of pork and to correlate the fusion data set with pork proportion in minced mutton.

\section{Materials and Methods}

2.1. Meat Samples. All the hind leg mutton samples detected by E-nose and E-tongue and used for determination of physical properties were obtained from the local logistics center for agricultural products, Hangzhou, China. The hind leg pork samples were purchased from Wal-Mart Stores, Hangzhou, China. The meat samples were brought to laboratory and stored at $-18^{\circ} \mathrm{C}$.

2.2. Sample Preparation. The fat and connective tissue were removed before samples were processed. The mutton and pork samples were cut into $1 \mathrm{~cm}^{3}$ cubic and minced for $2 \mathrm{~min}$ by a mincer, respectively. The adulterated mutton samples were made by mixing minced pork at levels of $0 \%$, $20 \%, 40 \%, 60 \%, 80 \%$, and $100 \%$ by weight with minced mutton and followed with mincing for $1 \mathrm{~min}$. After each sample was minced, the mincer was cleaned with detergent water and rinsing with distilled water, to prevent from cross interference of odour and flavor information. The adulterated meat samples were brought to room temperature before being detected by E-nose and E-tongue.

For detection of E-nose, the optimized detection parameters were as follows: $10 \mathrm{~g}$ of the minced meat was placed in a beaker of $250 \mathrm{~mL}$ at the temperature of $25^{\circ} \mathrm{C} \pm 3^{\circ} \mathrm{C}$, and the beaker was sealed by plastic for a headspace generation time of $30 \mathrm{~min}$. The headspace gas was detected by E-nose.

For detection by E-tongue, the optimized detection parameters were as follows: the taste substances of minced meat samples were extracted using potassium chloride solution $(0.1 \mathrm{~mol} / \mathrm{L})$ for 30 minutes at $4^{\circ} \mathrm{C}$ with a shaking (SKY$2112 \mathrm{~B}$, SUKUN, China) rate of $1500 \mathrm{rpm}$. The mixture was passed through the filter paper, and the supernatant was measured by E-tongue.

2.3. Detection Procedures of Electronic Nose (E-Nose) and Electronic Tongue (E-Tongue). To collect the odour fingerprint of the adulterated mutton, an E-nose of PEN 2 (Airsense Corporation, Germany) was used. The E-nose system consisted of three parts: the first is the sampling apparatus, the second is the detector unit containing of a sensor array of 10 different metal oxide sensors, and the third is pattern recognition software of Win Muster v.1.6. The nomenclature and characteristics of the 10 metal oxide sensors are listed in Table 1. It shows that each sensor has a certain degree of affinity towards specific chemical or volatile compounds.

For detection of adulterated mutton by E-nose, the experimental condition described in our former research [13] was used. The headspace generated by $10 \mathrm{~g}$ of samples in a beaker of $250 \mathrm{~mL}$ was detected for $80 \mathrm{~s}$, at the flow rate of $200 \mathrm{~mL} \cdot \mathrm{min}^{-1}$.

The $\alpha$-Astree taste system was employed to detect the gustatory information of the adulterated mutton samples. The E-tongue system comprised three parts of the sensor array, the reference electrode of $\mathrm{Ag} / \mathrm{AgC}$, and the autosampler with a mechanical stirrer. In the sensor array, the sensors were made from silicon transistors with an organic 
TABLe 1: Sensors used and their main applications in PEN 2 electronic nose.

\begin{tabular}{|c|c|c|c|}
\hline Number in array & Sensor name & General description & Reference \\
\hline S1 & W1C-aromatic & Aromatic compounds & Toluene, $10 \mathrm{ppm}$ \\
\hline S2 & W5S-broad range & $\begin{array}{c}\text { Very sensitive, broad range sensitivity, react on nitrogen oxides, very } \\
\text { sensitive with negative signal }\end{array}$ & $\mathrm{NO}_{2}, 1 \mathrm{ppm}$ \\
\hline S3 & W3C-aromatic & Ammonia, used as a sensor for aromatic compounds & Benzene, 1 ppm \\
\hline S4 & W6S-hydrogen & Mainly hydrogen, selectively (breath gases) & $\mathrm{H}_{2}, 100 \mathrm{ppb}$ \\
\hline S5 & W5C-arom-aliph & Alkanes, aromatic compounds, less polar compounds & Propane, $1 \mathrm{ppm}$ \\
\hline S6 & W1S-broad-methane & $\begin{array}{l}\text { Sensitive to methane (environment) ca, } 10 \mathrm{ppm} \text {, broad range, similar to no. } 8 \\
\text { Reacts on sulfur compounds, } \mathrm{H}_{2} \mathrm{~S} 0.1 \mathrm{ppm} \text {. Otherwise sensitive to many }\end{array}$ & $\mathrm{CH}_{4}, 100 \mathrm{ppm}$ \\
\hline S7 & W1W-sulfur-organic & $\begin{array}{c}\text { terpenes and sulfur organic compounds which are important for smell, } \\
\text { limonene, pyrazine }\end{array}$ & $\mathrm{H}_{2} \mathrm{~S}, 1 \mathrm{ppm}$ \\
\hline S8 & W2S-broad-alcohol & Detects alcohols, partially aromatic compounds, broad range & $\mathrm{CO}, 100 \mathrm{ppm}$ \\
\hline S9 & W2W-sulph-chlor & Aromatic compounds, sulfur organic compounds & $\mathrm{H}_{2} \mathrm{~S}, 1 \mathrm{ppm}$ \\
\hline S10 & W3S methane-aliph & Reacts on high concentration $>100 \mathrm{ppm}$, sometimes very selective (methane) & $\mathrm{CH}_{4}, 100 \mathrm{ppm}$ \\
\hline
\end{tabular}

TABLE 2: Sensors used in $\alpha$-Astree E-tongue and their thresholds $\left(\mathrm{mol} \cdot \mathrm{L}^{-1}\right)$.

\begin{tabular}{lcccccccc}
\hline $\begin{array}{l}\text { Basic } \\
\text { taste }\end{array}$ & $\begin{array}{c}\text { Taste } \\
\text { substance }\end{array}$ & ZZ & BA & BB & CA & GA & HA & JB \\
\hline Sour & Citric acid & $10^{-7}$ & $10^{-6}$ & $10^{-7}$ & $10^{-7}$ & $10^{-7}$ & $10^{-6}$ & $10^{-6}$ \\
Salty & KCL & $10^{-7}$ & $10^{-4}$ & $10^{-4}$ & $10^{-5}$ & $10^{-4}$ & $10^{-4}$ & $10^{-4}$ \\
Sweet & Glucose & $10^{-7}$ & $10^{-4}$ & $10^{-7}$ & $10^{-7}$ & $10^{-4}$ & $10^{-4}$ & $10^{-4}$ \\
Bitter & Caffeine & $10^{-5}$ & $10^{-4}$ & $10^{-4}$ & $10^{-5}$ & $10^{-4}$ & $10^{-4}$ & $10^{-4}$ \\
Savoury & L-Arginine & $10^{-6}$ & $10^{-4}$ & $10^{-6}$ & $10^{-5}$ & $10^{-5}$ & $10^{-4}$ & $10^{-5}$ \\
\hline
\end{tabular}

coating that governs sensitivity and selectivity of each individual sensor $[35,36]$. The thresholds [35] of 7 sensors to 5 basic tastes [36] are shown in Table 2.

The E-tongue system was conditioned with the conditioning sequence with the solution of $0.01 \mathrm{~mol} / \mathrm{L} \mathrm{HCl}$ to determine if the measured values are stable and repeatable. The calibration sequence was followed to adjust the measured values in the measuring range of the instrument. In the end, the diagnostic sequence was conducted with solution of $0.01 \mathrm{~mol} / \mathrm{L} \mathrm{HCl}, \mathrm{NaCl}$, and MSG to determine whether the measured values conform to the discrimination performance characteristics predefined by Alpha MOS for each sensor type.

The detection procedure of E-tongue is as follows: for each sample, the measurement time was set to $120 \mathrm{~s}$, and the sensors were rinsed for $10 \mathrm{~s}$ in potassium chloride solution $(0.1 \mathrm{~mol} / \mathrm{L})$ before the detection of the next sample. All the adulterated samples were detected at room temperature with 20 duplications.

2.4. Data Fusion Method. Either in the view of odour or flavor, E-nose and E-tongue have showed high ability in discrimination of adulterated mutton. Aiming at the combination of odour or flavor and giving an overall sensory result, the data fusion methods of low-level and midlevel were employed for the fusion of E-nose and E-tongue data. The information of data fusion methods for E-nose and E-tongue is described in Table 3.

For the low-level fusion method, (1) the E-nose data (120 repetitions of meat samples $\times 10$ sensors of E-nose) and E-tongue data ( 120 repetitions of meat samples $\times 7$ sensors of E-tongue) were gathered together to form a new data matrix
TABle 3: Data fusion methods for E-nose and E-tongue.

\begin{tabular}{lcc}
\hline $\begin{array}{l}\text { Fusion } \\
\text { level }\end{array}$ & Methods & $\begin{array}{c}\text { No. of } \\
\text { parameters }\end{array}$ \\
\hline Low & $\begin{array}{c}\text { Normalization after combination } \\
\text { Feature extracted by step-LDA } \\
\text { Extraction of features by principle } \\
\text { components }\end{array}$ & 17 \\
\hline Midlevel & Combination of extracted principle \\
components & 16 \\
\hline
\end{tabular}

with 120 rows and 17 columns for each sample. For all the adulterated mutton samples, the combined data matrix had 120 rows and 17 columns. (2) The $[0,1]$ maximumminimum normalized processing was conducted after data combination. Data selected by stepwise LDA were conducted by SAS version 8 (SAS Institute Inc., Gary, USA) on combined data of E-tongue and E-nose. The selection of the variable starts with the largest value of the $F$ statistic ( $F$ value) and the probability of $F$ lower than 0.15 . The procedure was repeated with the unselected variables and ends when the probability of $F$ is higher than 0.15 . As a result, 16 variables were selected for further analysis. Sensor responses with small influence on the identification process were switched off in further analysis. (3) Data extracted by principle component analysis was applied on the directly combined data set of E-nose and E-tongue $(120 \times 17), 99.0 \%$ cumulative calibrated variance was acquired, and as a result, 9 PCs (99.20\%) were extracted.

For midlevel level fusion methods, principle component analysis was applied to extracted features of E-nose and E-tongue signals before combining. For reducing the dimension of data set by linear combination of primary variables into the unrelated comprehensive index, principal component analysis is often used as the data extraction method. In order to retain most information of the original data, $99.0 \%$ cumulative calibrated variance was acquired. As a result, 6 PCs (99.52\%) based on E-nose and 5 PCs (99.43\%) based on E-tongue were used to form the combination matrix.

2.5. Data Analysis. To evaluate the discrimination effects of different fusion methods for E-nose and E-tongue, 
discriminant analysis methods of canonical discriminant analysis (CDA) with linear discriminant function and quadratic discriminant function and Bayes discriminant analysis (BDA) were used. To build the predictive models for the proportions of pork in adulterated mutton with the best fusion data set, methods of multiple linear regression (MLR), partial least square analysis (PLS), and backpropagation neural network (BPNN) were used. Both the qualitative and quantitative methods were compared to find the better one. The SAS version 8 (SAS Institute Inc., Gary, USA) was used for data processing, and all the figures were plotted by OriginPro 8 (OriginLab, USA).

\section{Results and Discussion}

3.1. Sensor Array Response to Meat Odour and Taste. In order to study the changing trend of E-nose and E-tongue sensors to adulterated mutton samples, the sensor responses of E-nose and E-tongue to minced mutton adulterated with $40 \%$ of pork are given in Figures 1 and 2. For 10 sensors of E-nose, at the initial response phase of $0-40 \mathrm{~s}$, the responses of S2, S8, and S6 increased with the collection time, and it is in accordance with the results of Zhan [37] that mutton was rich in volatile compounds and large content of alcohol, aldehydes, and ketones. The responses of S3, S1, and S5 dropped, for the content of aromatic compounds. However, the responses of S7, S9, S4, and S10 stayed stable during $0-50 \mathrm{~s}$. The responses of S7 and S9 increased slightly after $50 \mathrm{~s}$, while the responses of S4 and S10 stayed unchanged, as the content of hydrogen and methane-aliph was low. The responses of S2 kept rising during the whole collection time as it is very sensitive and with broad range sensitivity. The other 9 sensors' responses stabilized after collection time of $60 \mathrm{~s}$. For further data analysis, 10 sensors responses at the $75^{\text {th }}$ s were used, forming a data set of $10 \times 20$ for 6 adulterated mutton samples.

The adulterated mutton sample was detected by E-tongue, and the typical responses of E-tongue taste sensors to minced mutton adulterated with $40 \%$ of pork are shown in Figure 2. Taste sensors need time to adapt to the soluble flavor substances in the sample extraction. It was observed that most sensors adapt to the taste within $10 \mathrm{~s}$, while BB and $\mathrm{BA}$ need longer time of $20 \mathrm{~s}$ and $100 \mathrm{~s}$. For data analysis, the stable response at the $120^{\text {th }} \mathrm{s}$ of 7 sensors of E-tongue responses was extracted, forming a data set of $7 \times 20$ for 6 adulterated mutton samples.

3.2. Comparison of E-Nose and E-Tongue Results on Discrimination of Mutton Adulteration. For adulterated mutton samples, the odour and flavor information were obtained by E-nose and E-tongue, respectively. The stable values at the $75^{\text {th }} \mathrm{s}$ of E-nose responses and the stable values of the $120^{\text {th }} \mathrm{s}$ responses of E-tongue were extracted and used. For discrimination of mutton samples adulterated with different proportions of pork, three discriminant analysis methods were used and the results were compared, including canonical discriminant analysis (CDA) with linear discriminant function, canonical discriminant analysis with

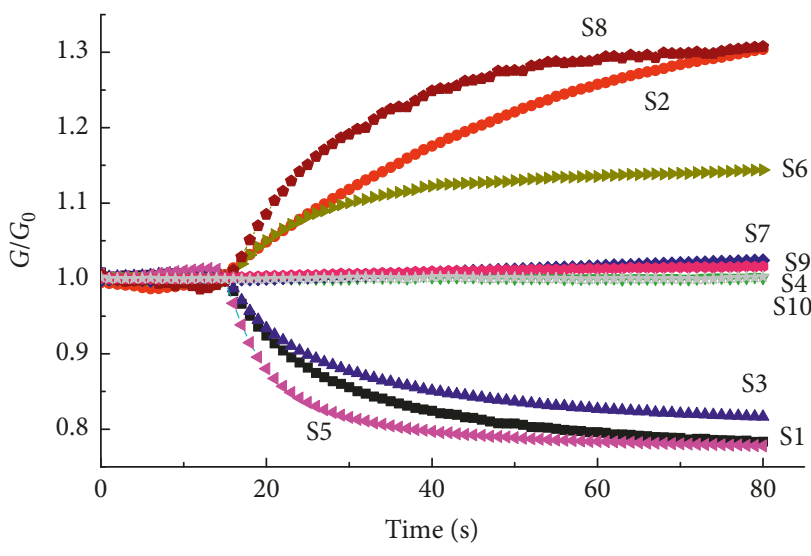

Figure 1: E-nose sensors' response curve to minced mutton adulterated with $40 \%$ of pork (S1: -; S2: •; S3: ^; S4: v; S5: ४; S6: •; S7: •; S8: •; S9: ; S10: $\star$ ).

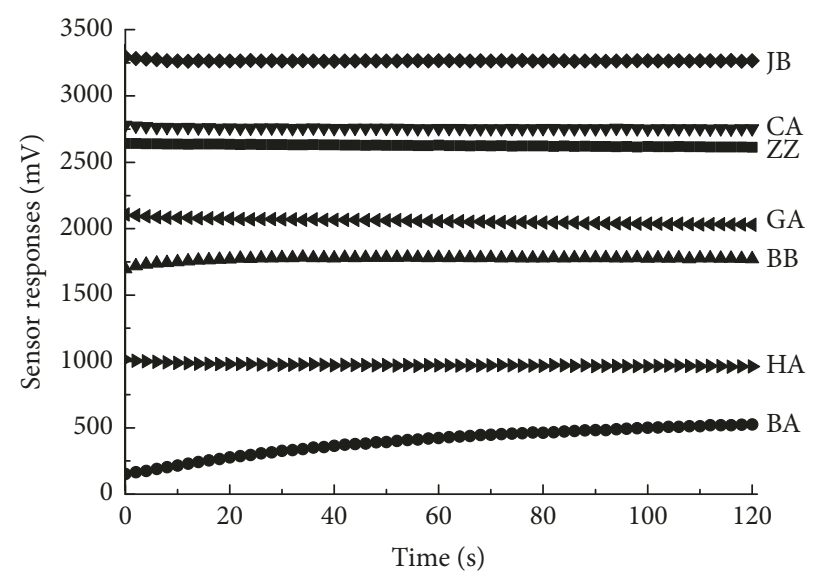

FIgURE 2: Typical response of E-tongue sensors to taste substance

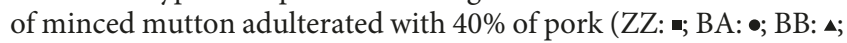
CA: $\mathbf{~ ; ~ G A : ~ « ; ~ H A : ~ • ; ~ J B : ~ • ) . ~}$

TABLE 4: Comparison results of discriminant analysis for responses of E-nose and E-tongue to minced mutton adulterated with pork.

\begin{tabular}{|c|c|c|c|}
\hline $\begin{array}{l}\text { Discrimination } \\
\text { method }\end{array}$ & Evaluation indices & $\begin{array}{l}\text { E-nose } \\
75^{\text {th }} \mathrm{s}\end{array}$ & $\begin{array}{c}\text { E-tongue } \\
120^{\text {th }} \mathrm{s}\end{array}$ \\
\hline \multirow{7}{*}{$\begin{array}{l}\text { Bayesian } \\
\text { discriminant } \\
\text { analysis } \\
\text { CDA-linear } \\
\text { discriminant } \\
\text { function } \\
\text { CDA-quadratic } \\
\text { discriminant } \\
\text { function }\end{array}$} & Number of misclassified & 7 & 0 \\
\hline & $\begin{array}{c}\text { Percentage of misclassified } \\
(\%)\end{array}$ & 5.83 & 0 \\
\hline & Number of misclassified & 13 & 3 \\
\hline & $\begin{array}{c}\text { Percentage of misclassified } \\
(\%)\end{array}$ & 10.83 & 2.5 \\
\hline & Number of misclassified & 13 & 2 \\
\hline & $\begin{array}{c}\text { Percentage of misclassified } \\
(\%)\end{array}$ & 10.83 & 1.67 \\
\hline & $\begin{array}{c}\text { Averaged percentage of } \\
\text { misclassified (\%) }\end{array}$ & 9.16 & 1.39 \\
\hline
\end{tabular}

quadratic discriminant function, and Bayes discriminant analysis. The discrimination results of E-nose and E-tongue are shown in Table 4. For E-nose, the percentage of misclassified was in the range of $5.83 \% \sim 10.85 \%$, and the best discrimination results were obtained by Bayes discriminant 
analysis. For E-tongue, the percentage of misclassified was in the range of $0 \sim 2.5 \%$, and the best discrimination results were obtained by Bayes discriminant analysis.

As a result, both E-nose and E-tongue could discriminate the adulteration of mutton with percentage of misclassified mutton samples adulterated with different proportions of pork lower than $11 \%$, and E-tongue showed higher ability in the adulteration detection of mutton. However, both E-nose and E-tongue detection showed only one aspect of sensory evaluation. Aiming at combination of odour or flavor and giving an overall sensory result, the data fusion methods of low-level and midlevel were employed for the fusion of E-nose and E-tongue data.

\subsection{Comparison of Fusion Methods for E-Nose and E-Tongue} Data on Adulteration Classification of Mutton Samples. As shown in Section 3.2, both E-nose and E-tongue could discriminate minced mutton adulterated with different proportions of pork with high accuracy. However, there were still few samples misclassified. The combination of E-nose and E-tongue was conducted using low-level and midlevel fusion methods. Discrimination methods of canonical discriminant analysis (CDA) with linear discriminant function, canonical discriminant analysis with quadratic discriminant function, and Bayes discriminant analysis were used, and the results were compared to get better identification results. The results obtained for each of the tasks are summarized in Table 5.

For low-level fusion, the following tasks were performed:

(1) E-nose and E-tongue data were combined directly, containing both useful and redundant information. The combined data set was analyzed by BDA and CDA with linear discriminant function and quadratic discriminant function after the normalization process. The results showed that only one sample was misclassified by CDA, with $99.45 \%$ correctly classified.

(2) After the combination of E-nose and E-tongue data, step-LDA was employed to eliminate redundant information, and the responses of sensor W1S of E-nose were removed. Compared with the directly combined data set, the discrimination results were same with the directly combined data set.

(3) Thus, PCA analysis was employed to analyze the combined data set of E-nose and E-tongue, and the first 9 principle components, containing $99.20 \%$ cumulative variance, were selected for discriminant analysis. The elimination of S6 leads to worse discrimination results, with 2 samples misclassified, with the average $1.11 \%$ percentage of misclassified mutton samples.

For the midlevel fusion, principal component analysis was used to extract features, reducing the dimension of data set by linear combination of primary variables into the unrelated comprehensive index, which is often used as the data extraction method. In order to retain most information of the original data, $99.0 \%$ cumulative calibrated variance was acquired. Thus, for E-nose, the first 6 principle components, containing $99.52 \%$ cumulative variance, and the first 5 principle components of E-tongue, containing 99.43\% cumulative variance, were selected to form a new data set comprising 11 components. The discrimination results were highly improved, with $100 \%$ correctly classified.

It can be seen that the data fusion processes showed good results as regards to the classification of minced mutton adulterated with different proportions of pork. The misclassified samples were lower than $1.67 \%$ in most cases. Compared with one detection method of E-nose or Etongue, the discrimination results were improved greatly with the fusion data of E-nose and E-tongue, especially for the fusion data set containing 6 PCs of E-nose and 5 PCs of E-tongue. The comparison showed that the results were always improved through combining taste and smell information in our work.

3.4. Adulteration Classification of Mutton Samples. To visualize the discrimination results, the results of CDA with linear discriminant function using data set of E-nose and E-tongue and fusion of E-nose and E-tongue were shown in Figure 3. For the CAN1 and CAN2, they explained 93.41\%, $93.09 \%, 90.24 \%, 89.99 \%, 88.68 \%$, and $89.52 \%$ of the total variance for different data sets with the value of $100 \%$, respectively. So, it can be inferred that the first two CANs can give most information of the data set. After applying the CDA method, the samples could be grouped into 6 clusters according to their pork proportions although there were few samples misclassified into other groups. Two discriminant functions showed good separation in the direction of CAN1 among 5 groups of minced meat containing mutton, and CAN1 increased with decreasing pork proportion. Furthermore, the pork samples scattered in the left side of the figure (Figure 3), which was far from the other groups. With different proportions of mutton, all minced mixed meat had the smell of mutton with diverse intensity, which could be used as an index in discrimination of adulteration.

For discrimination, samples in groups of $40 \%, 60 \%$, and $80 \%$ overlapped with each other when analyzed by E-nose (Figure 3(b)), and samples in groups of $40 \%$ and $20 \%$ overlapped with each other when analyzed by E-tongue with CDA (Figure 3(a)). When data fusion methods were used, the discrimination results of CDA were improved in the scatter plot of Figure 3 and Table 5, except that one or two samples were close to the adjacent groups. The best method was found to be the combination of principle components of each data set for fusion of E-nose and E-tongue data (6 PCs of E-nose and 5 PCs of E-tongue), with no sample misclassified by CDA.

For BDA, the discrimination results were same with the results obtained with E-tongue data only, better than with E-nose data. The discrimination results were not always improved using fusion data, which were similar with the results of Cosio at 2007 [38].

3.5. Rapid Characterisation of Pork Proportions in Minced Mutton. In order to establish the relationship between E-panel and pork proportions in minced mutton and to 
TABLE 5: Comparison results of discriminant analysis for different data fusion methods for responses of E-nose and E-tongue to minced mutton adulterated with pork.

\section{Original sensor fusion method}

Fusion

methods
Feature extraction

\section{Normalization}

Extraction of principle components
Combination of extracted data

Extraction of principle components

\begin{tabular}{|c|c|c|c|c|c|}
\hline & & & & & \\
\hline Bayesian & Number of misclassified & 0 & 0 & 0 & 0 \\
\hline discriminant analysis & Percentage of misclassified & 0 & 0 & 0 & 0 \\
\hline CDA-linear & Number of misclassified & $1(20 \%$ to $40 \%)$ & $1(20 \%$ to $40 \%)$ & $2(40 \%$ to $20 \%)$ & 0 \\
\hline discriminant function & Percentage of misclassified & $0.83 \%$ & $0.83 \%$ & $1.67 \%$ & 0 \\
\hline CDA-quadratic & Number of misclassified & $1(40 \%$ to $20 \%)$ & $1(40 \%$ to $20 \%)$ & $\begin{array}{c}2(1 \text { was } 40 \% \text { to } 20 \% \text {; } \\
1 \text { was } 20 \% \text { to } 40 \%)\end{array}$ & 0 \\
\hline aiscriminant & Percentage of misclassified & $0.83 \%$ & $0.83 \%$ & $1.67 \%$ & 0 \\
\hline Averaged & & $0.55 \%$ & $0.55 \%$ & $1.11 \%$ & 0 \\
\hline
\end{tabular}

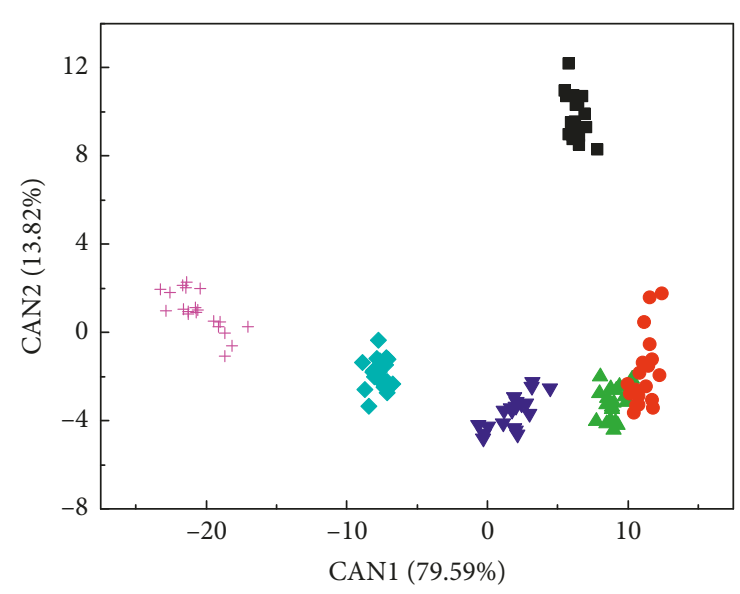

Proportion
+ Pork
- $80 \%$
จ $60 \%$
$\Delta 40 \%$
- $20 \%$
- Mutton

(a)

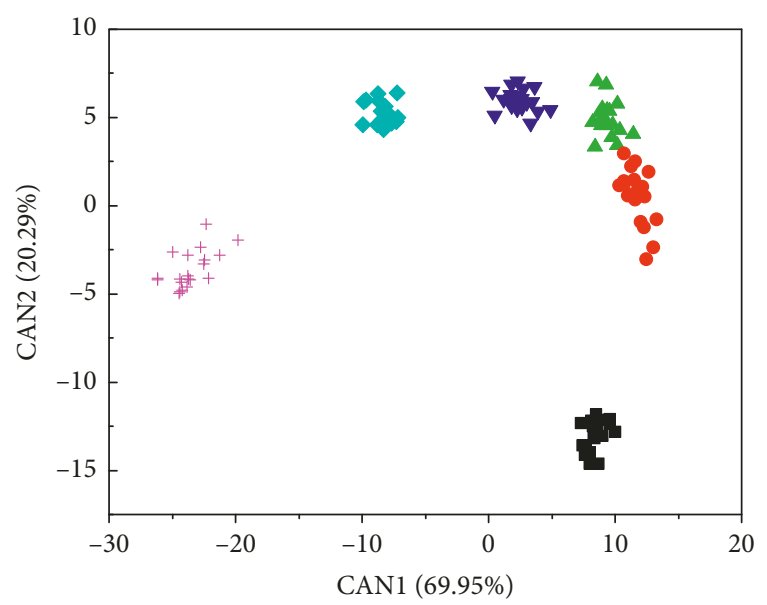

Proportion
+ Pork
- $80 \%$
- $40 \%$
- $20 \%$
จ $60 \%$

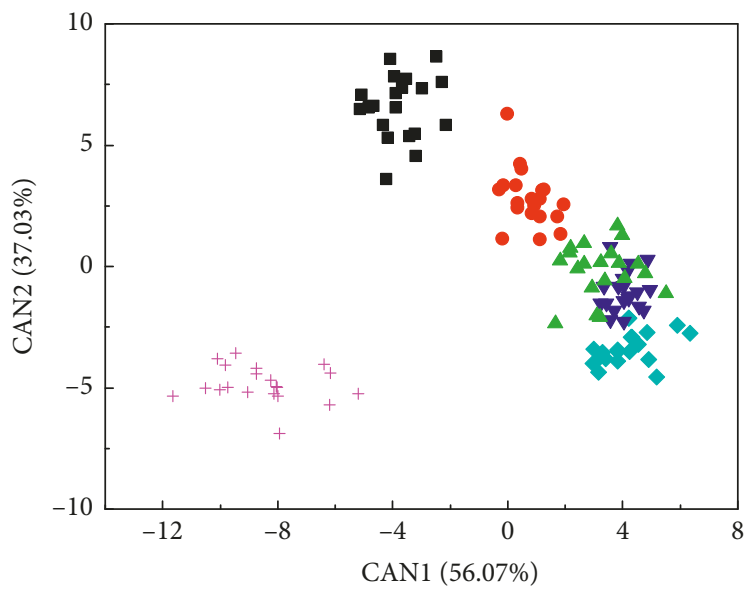

Proportion

+ Pork

- $80 \%$

- $60 \%$

$40 \%$

- $20 \%$

- Mutton

(b)

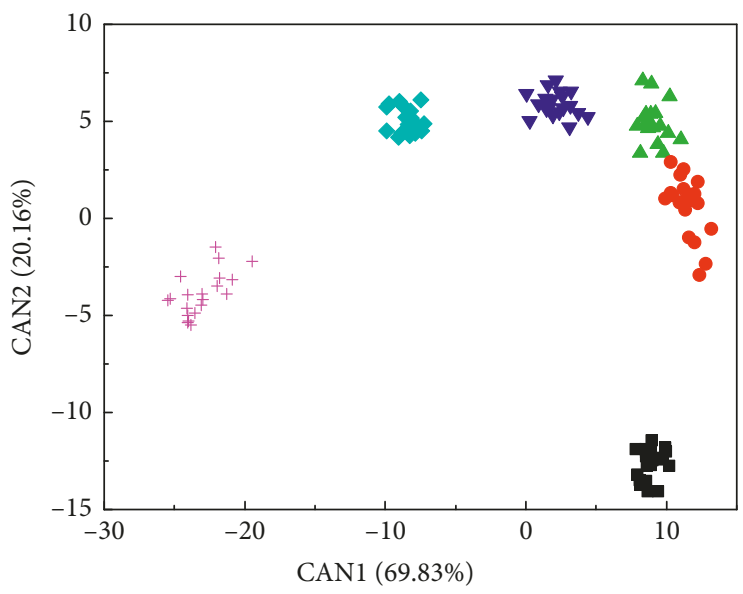

Proportion
+ Pork
- $40 \%$
- $80 \%$
- $20 \%$
> $60 \%$
- Mutton

(c)

(d)

Figure 3: Continued. 

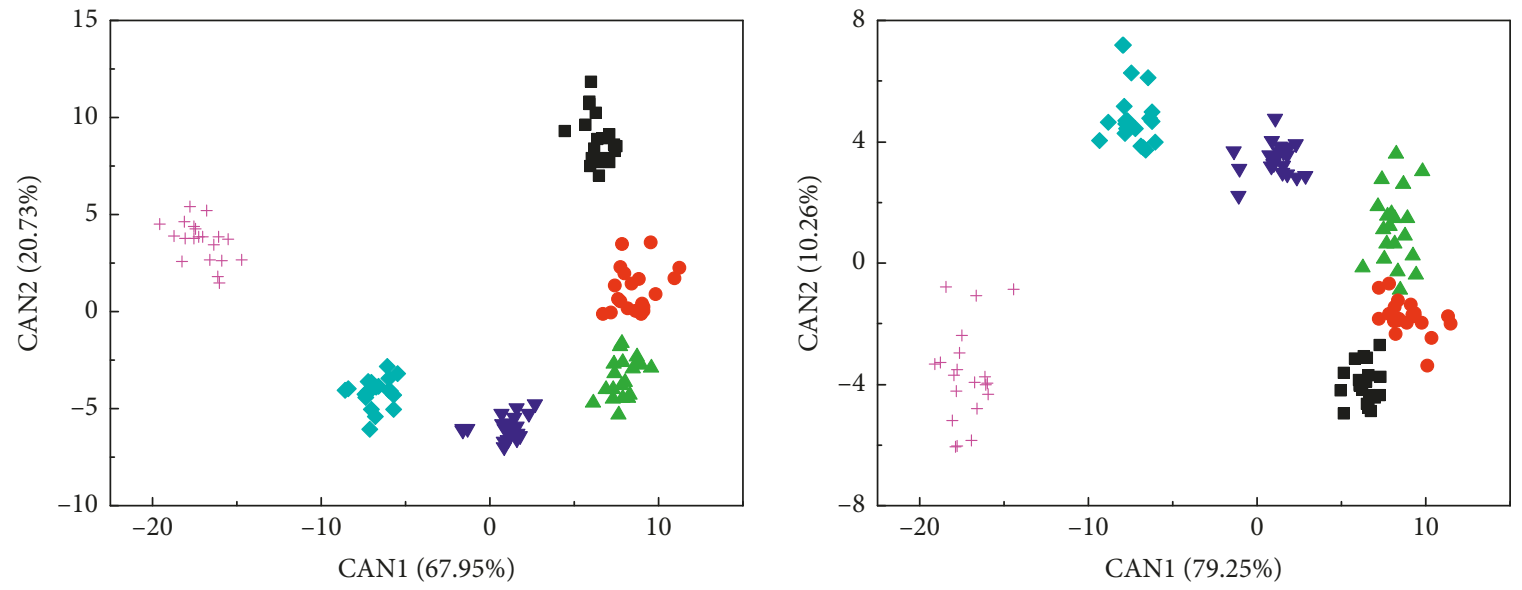

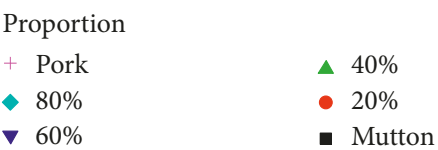

(e)

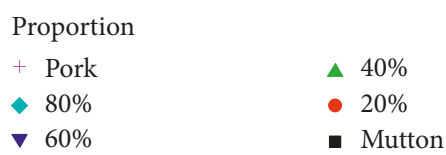

(f)

Figure 3: Scatter plot of CDA with linear discriminant function: (a) E-tongue; (b) E-nose; (c) original sensor fusion of E-tongue and E-nose; (d) features extracted by stepwise LDA on original sensor fusion data; (e) combination of features of E-nose and E-tongue extracted by PCA; (f) features extracted by PCA on original sensor fusion data.

classify meat samples according to their proportions of pork, analytical methods of partial least square (PLS), multiple linear regressions (MLR), and backpropagation neural network (BPNN) were used, and the results were compared to the best prediction model.

Here, the fusion data set, which contains 6 PCs of E-nose and 5 PCs of E-tongue, was used as input data to build the predictive models. The samples used for the training data set and testing data set were randomly selected in the experiment. 96 meat samples were used to build the predictive model for the proportions of pork adulterated into minced mutton, and 24 meat samples were used to validate the model. The performance of the model was evaluated by correlation coefficient $\left(R^{2}\right)$ and root mean square error (RMSE) between experimental values and predicted values.

For BPNN, the experimental design was completely randomized with each sample as an experimental unit. The architecture of the artificial neural network chosen was $N \times(2 N+1) \times M$ three-layered backpropagation, where $N$ is the number of inputs and $M$ is the number of outputs. The three layers are as follows: the input layer was designed as 11 neurons according to the sensor array fused by combination of 6 PCs of E-nose and 5 PCs of E-tongue; 1 hidden layer; and the output layer had 6 neurons for different proportions of pork in minced mutton.

The results obtained by the training model of BPNN showed that all the training and testing samples were correctly classified according to their proportions of pork. And the correlations between the observed and predicted proportions of pork in minced mutton were higher than 0.99 (Table 6 and Figure 4) both for the training and testing sets. The low errors of prediction and the high correlation of the BPNN model suggested that the fusion of E-nose and
E-tongue can be successfully applied in the determination of the adulteration detection of pork in minced mutton.

The multivariate projection method of PLS, with the leave-one-out technique, was applied to model the relationship between dependent variables and independent variables. The accuracy was estimated using the parameters ( $R^{2}$ and RMSE) obtained from the fitted equation. As shown in Table 6 and Figure 4, good correlations of calibration were found between E-nose data and content of pork with a determination of coefficient $R^{2}=0.9791$. When the model was applied to predict the other 24 samples, similar results could be found, and good prediction results for content of pork with the coefficient $R^{2}$ of 0.9758 was obtained. The PLSE-tongue methods have been proved to be suitable for prediction of chemical parameters $[39,40]$. So, pork proportion in minced mutton could be determined simultaneously by PLS using the E-tongue data in this work.

The relationship between fusion signals of E-nose and E-tongue and the pork content in adulterated mutton was studied by the MLR algorithm, and the results are shown in Table 6 and Figure 4. A linear correlation between fusion signals and pork content were found, with $R^{2}=0.9746$ and $\mathrm{RMSE}=5.04 \%$ for the training set. And when it was used in the testing data set, high predictive ability was found with $R^{2}=0.9755$ and $\mathrm{RMSE}=5.52 \%$.

In conclusion, excellent prediction ability was found using PLS, MLR, and BPNN for the prediction of pork proportion in minced mutton with $R^{2}$ higher than 0.9609 and RMSE lower than $10.94 \%$. Compared with predictive results obtained by E-nose, BPNN, MLR, and PLS improved the predictive results of $R^{2}$ or RMSE value, indicating that the combination of more information could lead to better results. However, compared with E-tongue, the predictive 
TABLE 6: Prediction of pork proportion in minced mutton by signals of E-nose, E-tongue, and the combined data set of E-nose and E-tongue.

\begin{tabular}{|c|c|c|c|c|c|}
\hline \multirow{2}{*}{ Data used } & \multirow{2}{*}{ Methods } & \multicolumn{2}{|c|}{ Calibration } & \multicolumn{2}{|c|}{ Validation } \\
\hline & & $R^{2}$ & RMSEC (\%) & $R^{2}$ & RMSEP (\%) \\
\hline \multirow{3}{*}{ E-nose (variables selected by stepwise LDA) } & PLS & 0.9609 & 6.72 & 0.9092 & 10.94 \\
\hline & MLR & 0.9609 & 6.72 & 0.91 & 10.94 \\
\hline & BPNN & 0.9886 & 3.78 & 0.9762 & 5.26 \\
\hline \multirow{3}{*}{ E-tongue } & PLS & 0.987 & 3.90 & 0.9823 & 7.96 \\
\hline & MLR & 0.9869 & 3.90 & 0.9827 & 7.52 \\
\hline & BPNN & 0.9950 & 2.44 & 0.9956 & 2.23 \\
\hline \multirow{3}{*}{ Fusion of E-nose and E-tongue } & PLS & 0.9791 & 4.90 & 0.9758 & 5.37 \\
\hline & MLR & 0.9746 & 5.04 & 0.9755 & 5.52 \\
\hline & BPNN & 0.9966 & 2.23 & 0.9928 & 3.01 \\
\hline
\end{tabular}

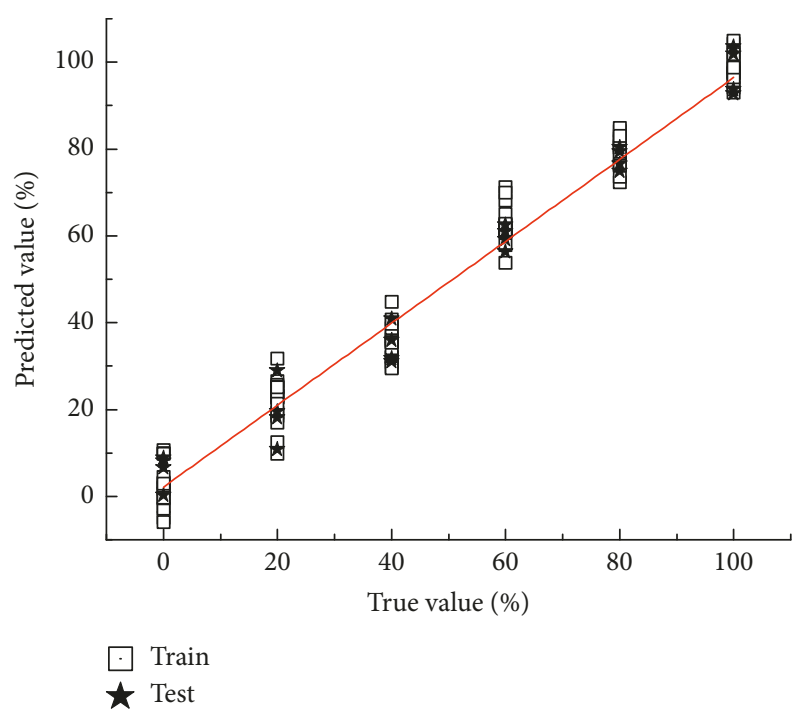

(a)

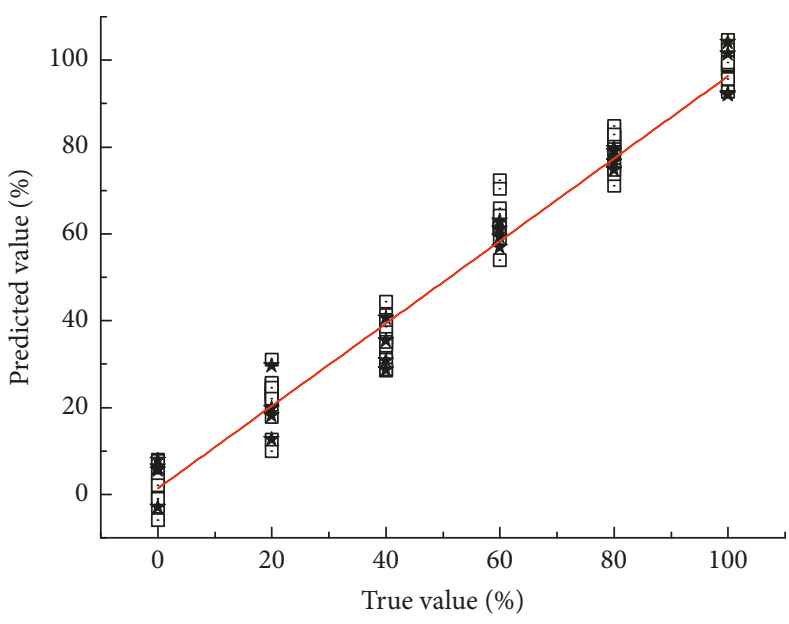

$\square$ Train $\star$ Test

(b)

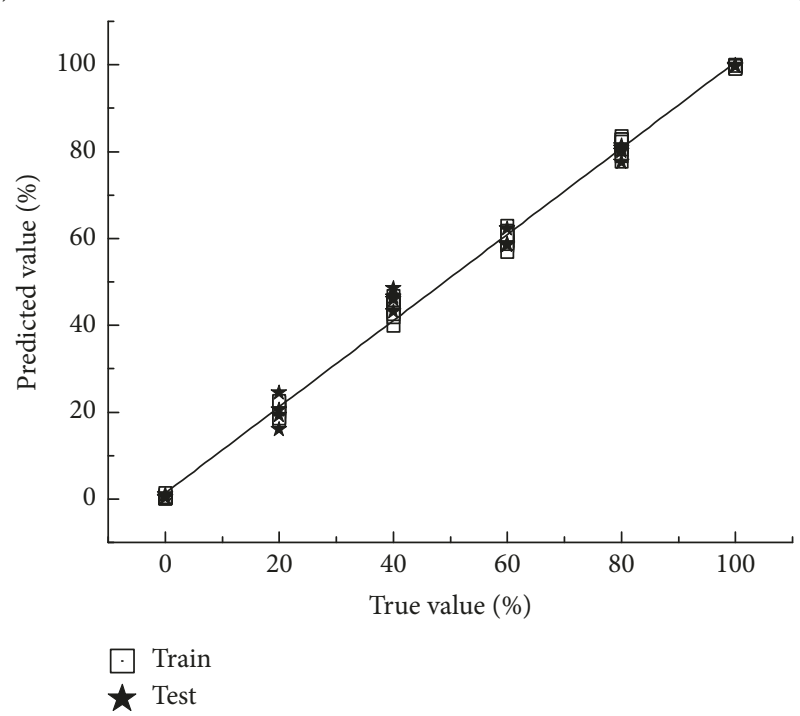

(c)

FIGURE 4: Regression between the expected and true proportion of pork in minced mutton using combined data set of E-nose and E-tongue: (a) PLS; (b) MLR; (c) BPNN. 
results of PLS and MLR were similar or even lower with data combined. The best predictive results were found by the BPNN model with the combined data set, with high correlation (higher than 0.99) for the training and testing subsets.

\section{Conclusions}

The data fusion methods (low-level and midlevel) proved the effective combination of E-nose and E-tongue for the authentication of mutton accordingly to the proportion of pork. Of the two levels of fusion methods, midlevel level fusion, using principle component extraction, exhibited good discrimination results with discrimination methods of CDA, with all samples correctly classified, same with BDA using E-tongue data for the fusion data with any methods. Strong correlation was observed between the E-panel system and proportion of pork in minced mutton, and BPNN was the most suitable one for the prediction of pork proportion. The results obtained by this study are promising in terms of further development of rapid detection method based on E-panel systems for meat authentication in the meat industry.

\section{Data Availability}

The statistical data used to support the findings of this study are included within the supplementary information file.

\section{Conflicts of Interest}

The authors declare that they have no conflicts of interest.

\section{Acknowledgments}

The authors gratefully acknowledge the financial support from the National Natural Science Foundation of China (31560477 and 31071548) and Gansu Province Science and Technology Funding Plan through projects (18JR3RA371 and 17YF1WA166).

\section{Supplementary Materials}

The statistical data used to support the findings of this study are included within the supplementary information files. (Supplementary Materials)

\section{References}

[1] K. Paulos, S. Rodrigues, A. F. Oliveira, A. Leite, E. Pereira, and A. Teixeira, "Sensory characterization and consumer preference mapping of fresh sausages manufactured with goat and sheep meat," Journal of Food Science, vol. 80, no. 7, pp. S1568-S1573, 2015.

[2] D. Cozzolino and I. Murray, "Identification of animal meat muscles by visible and near infrared reflectance spectroscopy," LWT-Food Science and Technology, vol. 37, no. 4, pp. 447-452, 2004.

[3] M. Kamruzzaman, D.-W. Sun, G. Elmasry, and P. Allen, "Fast detection and visualization of minced lamb meat adulteration using NIR hyperspectral imaging and multivariate image analysis," Talanta, vol. 103, no. 2, pp. 130-136, 2013.

[4] X. Tian, J. Wang, R. Shen, Z. Ma, and M. Li, "Discrimination of pork/chicken adulteration in minced mutton by electronic taste system," International Journal of Food Science \& Technology, vol. 54, no. 3, pp. 670-678, 2019.

[5] B. Druml, S. Grandits, W. Mayer, R. Hochegger, and M. Cichna-Markl, "Authenticity control of game meat products-A single method to detect and quantify adulteration of fallow deer (Dama dama), red deer (Cervus elaphus) and sika deer (Cervus nippon) by real-time PCR," Food Chemistry, vol. 170, pp. 508-517, 2015.

[6] M. M. Rahman, M. E. Ali, S. B. A. Hamid, S. Mustafa, U. Hashim, and U. K. Hanapi, "Polymerase chain reaction assay targeting cytochrome $\mathrm{b}$ gene for the detection of dog meat adulteration in meatball formulation," Meat Science, vol. 97, no. 4, pp. 404-409, 2014.

[7] Q. Rao and Y.-H. P. Hsieh, "Evaluation of a commercial lateral flow feed test for rapid detection of beef and sheep content in raw and cooked meats," Meat Science, vol. 76, no. 3, pp. 489-494, 2007.

[8] A. M. A. Di Giuseppe, N. Giarretta, M. Lippert, V. Severino, and A. Di Maro, "An improved UPLC method for the detection of undeclared horse meat addition by using myoglobin as molecular marker," Food Chemistry, vol. 169, pp. 241-245, 2015.

[9] M. Nurjuliana, Y. B. Che Man, D. Mat Hashim, and A. K. S. Mohamed, "Rapid identification of pork for halal authentication using the electronic nose and gas chromatography mass spectrometer with headspace analyzer," Meat Science, vol. 88, no. 4, pp. 638-644, 2011.

[10] N. Morsy and D.-W. Sun, "Robust linear and non-linear models of NIR spectroscopy for detection and quantification of adulterants in fresh and frozen-thawed minced beef," Meat Science, vol. 93, no. 2, pp. 292-302, 2013.

[11] M. Zhao, G. Downey, and C. P. O'Donnell, "Detection of adulteration in fresh and frozen beefburger products by beef offal using mid-infrared ATR spectroscopy and multivariate data analysis," Meat Science, vol. 96, no. 2, pp. 1003-1011, 2014.

[12] A. I. Ropodi, D. E. Pavlidis, F. Mohareb, E. Z. Panagou, and G.-J. E. Nychas, "Multispectral image analysis approach to detect adulteration of beef and pork in raw meats," Food Research International, vol. 67, pp. 12-18, 2015.

[13] X. Tian, J. Wang, and S. Cui, "Analysis of pork adulteration in minced mutton using electronic nose of metal oxide sensors," Journal of Food Engineering, vol. 119, no. 4, pp. 744-749, 2013.

[14] J. Han, L. Huang, Z. Gu, S. Tian, and S. Deng, "Evaluation of meat quality and freshness based on the electronic tongue," Journal of Chinese Institute of Food Science and Technology, vol. 8, no. 3, pp. 125-132, 2008, in Chinese.

[15] P. Wang, Y. Zhang, X. Xu, and G. Zhou, "Discrimination on chicken meat products with electronic tongue," in Proceedings of the Chinese Society of Agricultural Engineering, 2011, in Chinese.

[16] A. L. A. Rudnitskaya, B. Seleznev, and Y. Vlasov, "Recognition of liquid and flesh food using an 'electronic tongue," International Journal of Food Science and Technology, vol. 37, no. 4, pp. 375-385, 2002.

[17] M. García, M. Aleixandre, J. Gutiérrez, and M. C. Horrillo, "Electronic nose for wine discrimination," Sensors and Actuators B: Chemical, vol. 113, no. 2, pp. 911-916, 2006.

[18] T. Horrillo, S. Marco, A. Perera et al., "Potato creams recognition from electronic nose and tongue signals: feature 
extraction/selection and RBF neural networks classifiers," in Proceedings of the 5th Seminar on Neural Network Applications in Electrical Engineering, 2000 (NEUREL 2000), pp. 69-74, IEEE, Belgrade, Serbia, September 2000.

[19] Z. Haddi, M. Boughrini, S. Ihlou et al., "Geographical classification of Virgin Olive Oils by combining the electronic nose and tongue," in Proceedings of the Sensors, 2012 IEEE, pp. 1-4, IEEE, Taipei, Taiwan, October 2012.

[20] Z. Haddi, H. Alami, N. El Bari et al., "Electronic nose and tongue combination for improved classification of Moroccan virgin olive oil profiles," in Food Research International, M. Luz Méndez Méndez, Ed., pp. 277-289, Academic Press, Cambridge, MA, USA, 2016.

[21] C. Apetrei, M. Ghasemi-Varnamkhasti, and I. Mirela Apetrei, Olive Oil and Combined Electronic Nose and Tongue, Electronic Noses and Tongues in Food Science, M. Luz Rodríguez Méndez, Ed., pp. 277-289, Academic Press, Cambridge, MA, USA, 2016.

[22] D. Huo, Y. Wu, M. Yang, H. Fa, X. Luo, and C. Hou, "Discrimination of Chinese green tea according to varieties and grade levels using artificial nose and tongue based on colorimetric sensor arrays," Food Chemistry, vol. 145, pp. 639-645, 2014.

[23] R. Banerjee, B. Tudu, L. Shaw, A. Jana, N. Bhattacharyya, and R. Bandyopadhyay, "Instrumental testing of tea by combining the responses of electronic nose and tongue," Journal of Food Engineering, vol. 110, no. 3, pp. 356-363, 2012.

[24] M. L. Rodriguez-Mendez, A. A. Arrieta, V. Parra et al., "Fusion of three sensory modalities for the multimodal characterization of red wines," IEEE Sensors Journal, vol. 4, no. 3, pp. 348-354, 2004.

[25] Z. Haddi, S. Mabrouk, M. Bougrini et al., "E-nose and e-tongue combination for improved recognition of fruit juice samples," Food Chemistry, vol. 150, pp. 246-253, 2014.

[26] S. Qiu, J. Wang, and L. Gao, "Qualification and quantisation of processed strawberry juice based on electronic nose and tongue," LWT-Food Science and Technology, vol. 60, no. 1, pp. 115-123, 2015.

[27] M. Laureati, S. Buratti, A. Bassoli, G. Borgonovo, and E. Pagliarini, "Discrimination and characterisation of three cultivars of Perilla frutescens by means of sensory descriptors and electronic nose and tongue analysis," Food Research International, vol. 43, no. 4, pp. 959-964, 2010.

[28] W. Dong, J. Zhao, R. Hu, Y. Dong, and L. Tan, "Differentiation of Chinese robusta coffees according to species, using a combined electronic nose and tongue, with the aid of chemometrics," Food Chemistry, vol. 229, pp. 743-751, 2017.

[29] L. Gil-Sánchez, J. Soto, R. Martínez-Máñez, E. Garcia-Breijo, J. Ibáñez, and E. Llobet, "A novel humid electronic nose combined with an electronic tongue for assessing deterioration of wine," Sensors and Actuators A: Physical, vol. 171, no. 2, pp. 152-158, 2011.

[30] C. Di Natale, R. Paolesse, A. Macagnano et al., "Electronic nose and electronic tongue integration for improved classification of clinical and food samples," Sensors and Actuators B: Chemical, vol. 64, no. 1-3, pp. 15-21, 2000.

[31] C. Apetrei, I. M. Apetrei, S. Villanueva, J. A. de Saja, F. Gutierrez-Rosales, and M. L. Rodriguez-Mendez, "Combination of an e-nose, an e-tongue and an e-eye for the characterisation of olive oils with different degree of bitterness," Analytica Chimica Acta, vol. 663, no. 1, pp. 91-97, 2010.

[32] X. Hong and J. Wang, "Detection of adulteration in cherry tomato juices based on electronic nose and tongue: comparison of different data fusion approaches," Journal of Food Engineering, vol. 126, pp. 89-97, 2014.

[33] A. R. D. Rosa, F. Leone, F. Cheli, and V. Chiofalo, "Fusion of electronic nose, electronic tongue and computer vision for animal source food authentication and quality assessment-a review," Journal of Food Engineering, vol. 210, pp. 62-75, 2017.

[34] E. Borràs, J. Ferré, R. Boqué, M. Mestres, L. Aceña, and O. Busto, "Data fusion methodologies for food and beverage authentication and quality assessment-A review," Analytica Chimica Acta, vol. 891, pp. 1-14, 2015.

[35] Alpha MOS, Technical Note T-P-02, Similarities between Electronic Tongue and Human Tongue, Alpha MOS, Toulouse, France, 2005.

[36] Alpha MOS, Technical Note T-SAS-01, Electrochemical Sensors, Alpha MOS, Toulouse, France, 2002.

[37] P. Zhan, H. Tian, W. Li, Y. Ma, and X. Zhang, "Establishment of mutton characteristics flavor fingerprint," Modern Food Science and Technology, vol. 29, no. 10, pp. 2522-2527, 2013.

[38] M. S. Cosio, D. Ballabio, and C. S. Gigliotti, "Evaluation of different storage conditions of extra virgin olive oils with an innovative recognition tool built by means of electronic nose and electronic tongue," Food Chemistry, vol. 101, no. 2, pp. 485-491, 2007.

[39] C. Apetrei, I. M. Apetrei, I. Nevares et al., "Using an e-tongue based on voltammetric electrodes to discriminate among red wines aged in oak barrels or aged using alternative methods," Electrochimica Acta, vol. 52, no. 7, pp. 2588-2594, 2007.

[40] A. De Saja, E. Polshin, D. Kirsanov et al., "Instrumental measurement of beer taste attributes using an electronic tongue," Analytica Chimica Acta, vol. 646, no. 1-2, pp. 111-118, 2009. 


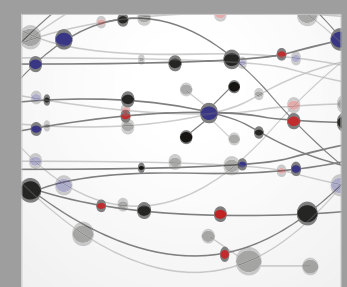

The Scientific World Journal
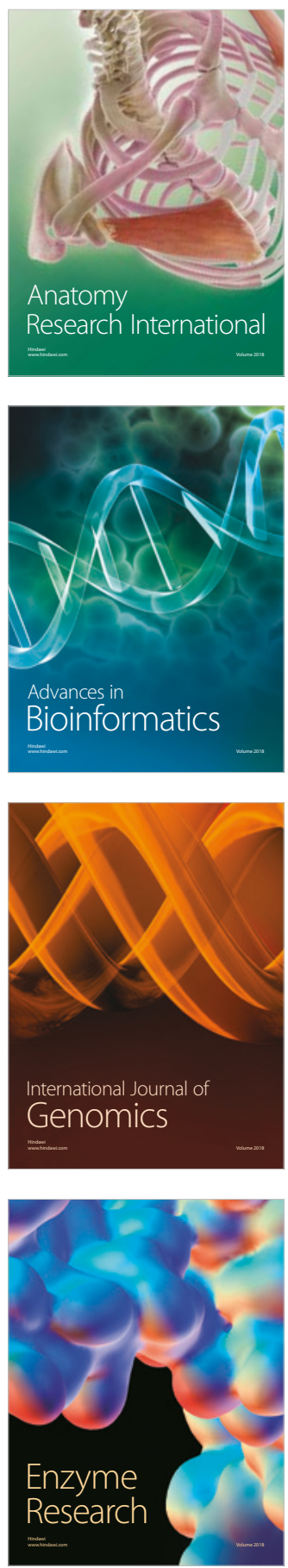
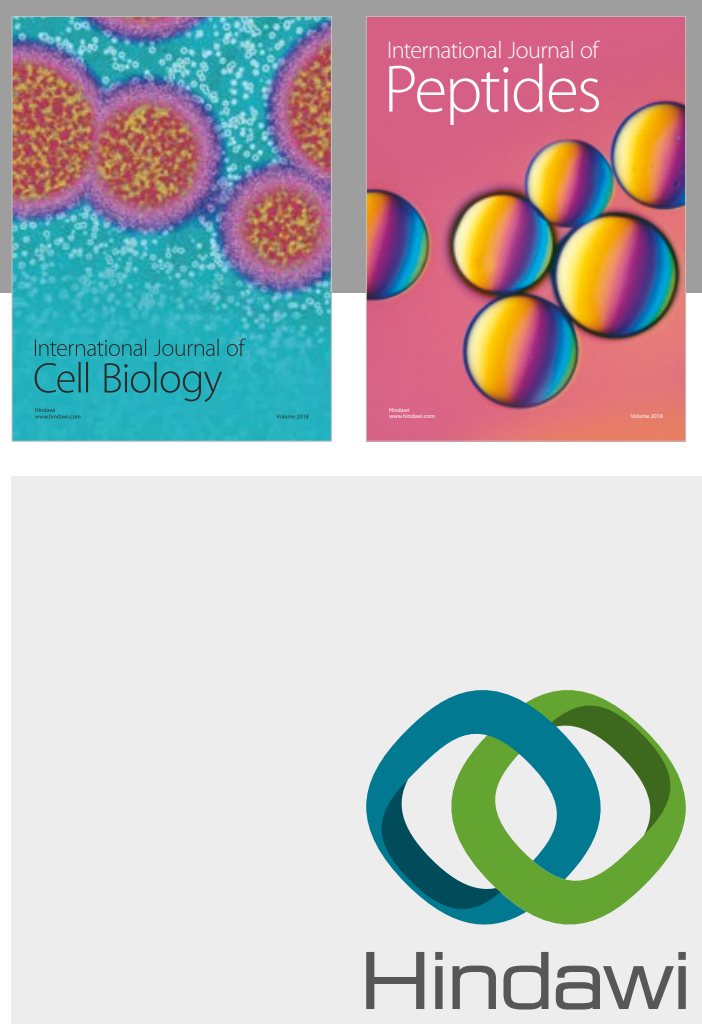

Submit your manuscripts at

www.hindawi.com
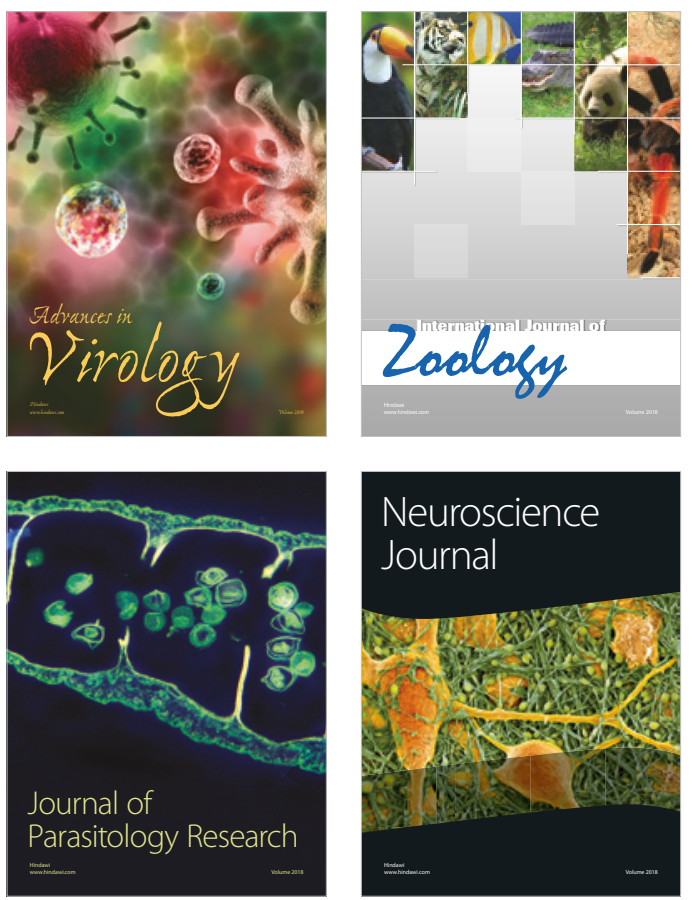
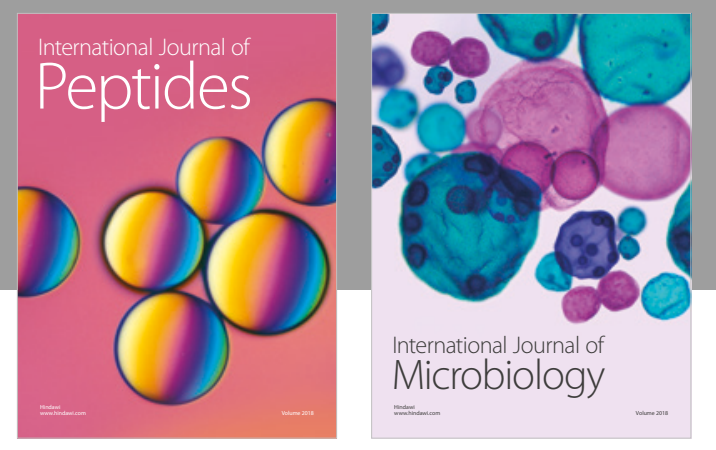

nternational Journal of Microbiology
Journal of
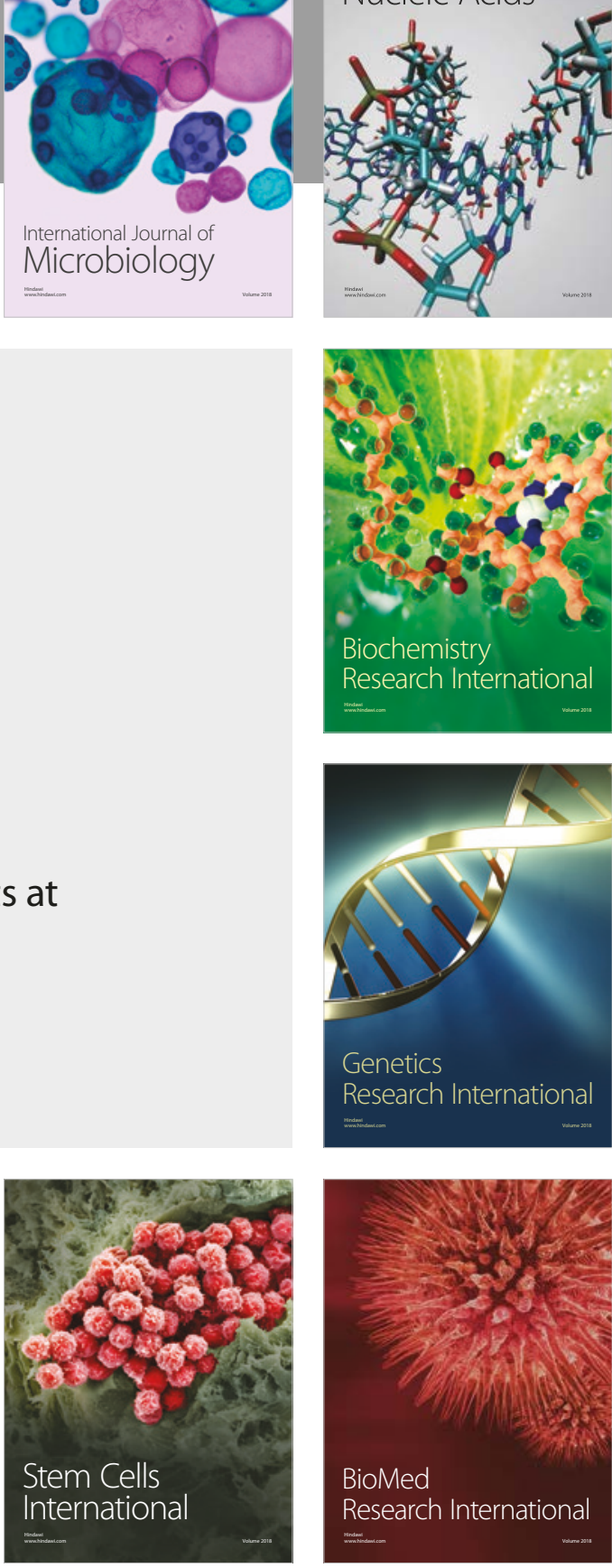
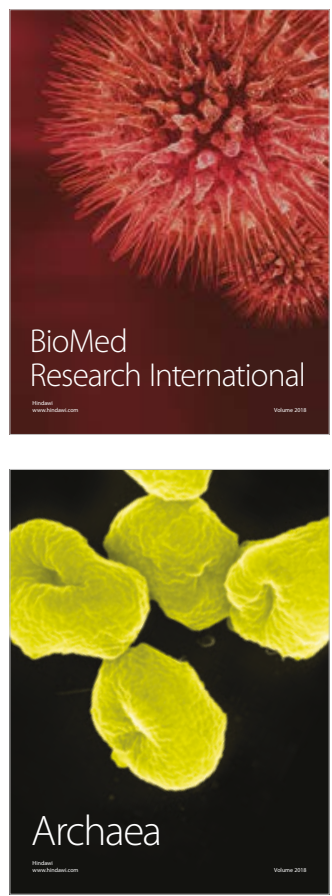